\title{
Análisis de la contratación directa en la causal de ciencia, tecnología e innovación bajo los principios de planeación y selección objetiva ${ }^{1}$
}

\section{Analysis of direct contracting in the cause of science, technology and innovation under the principles of planning and objective selection}

Katherine Buendia Franco*

\section{Resumen}

Esta investigación consiste en el análisis de la seguridad jurídica que existe en la modalidad de contratación directa bajo la causal de ciencia, tecnología e innovación en desarrollo de los principios de planeación y selección objetiva, a través de una revisión documental divida en dos secciones; por un lado, se delimita el principio de planeación en complemento con el principio de selección objetiva en el marco de la contratación estatal; posteriormente, se conceptualiza la contratación directa bajo la causal de Ciencia, Tecnología e Innovación, y se concluye con un análisis de la aplicación de la causal de la contratación directa bajo el principio de selección objetiva. Así se determina que no se puede aplicar la causal sin realizar un análisis a la regulación taxativa establecida por el legislador para las actividades

1 Artículo de investigación.

* Abogada de la Pontificia Universidad Javeriana de Bogotá; especialista en Derecho Público, Ciencia y Sociología Política de la Universidad Externado de Colombia; magíster en Derecho Contractual Público y Privado en la Universidad Santo Tomás de Bogotá. 
de ciencia, tecnología e innovación. Por lo tanto, no se permite una interpretación o analogía de estas actividades por parte del ejecutor, quien está obligado a realizar un estudio profundo de las formalidades y procedimientos a seguir de contemplar esta modalidad. El método de investigación aplicado a este documento es el cualitativo con un enfoque hermenéutico-crítico, el cual se realizó a través de la estrategia de revisión documental.

\section{Palabras clave}

Contratación directa, ciencia, tecnología e innovación, selección objetiva, planeación y contratación pública.

\section{Abstract}

This research consists of the analysis of the legal security that exists in the direct contracting modality under the cause of science, technology and innovation in development of the principles of planning and objective selection through a documentary review divided into two sections; On the one hand, the planning principle is delimited in addition to the objective selection principle in the framework of state contracting to later conceptualize direct contracting under the cause of Science, Technology and Innovation. To conclude with an analysis of the application of the cause of direct contracting under the principle of objective selection. Thus it is determined that the cause cannot be applied without carrying out an analysis of the restrictive regulation established by the legislator for science, technology and innovation activities. Therefore, an interpretation or analogy of these activities by the executing agency is not allowed, they are obliged to carry out an in-depth study of the formalities and procedures to be followed in considering this modality. The research method applied to this document is qualitative with a hermeneutical-critical approach, which was carried out through the document review strategy. 


\section{Keywords}

Direct contracting, Science, technology and innovation, Objective selection, Planning, Public contracting.

\section{Introducción}

Dentro del campo de la contratación estatal, se ha identificado la necesidad del Estado de reglamentar la adquisición de bienes y servicios, razón por la cual se creó la normatividad que establece los parámetros generales para la consecución de esta finalidad. La generalidad de la contratación es la que permite presentar oferta a los interesados en realizar la prestación de un servicio o la venta de un bien, pero partiendo de la planeación de una necesidad específica del Estado. A través de los años se han afinado los criterios de selección objetiva y se han introducido diferentes modalidades que permiten la participación de los particulares en los procesos de selección, tales como la licitación pública, la selección abreviada, el concurso de méritos y la contratación directa.

En concordancia con los fines del Estado, a partir de la Constitución Política de 1991 se ha identificado la modalidad de licitación como la más acorde con estos, debido a que permite una gran participación de interesados; sin embargo, por su regulación específica y sus extensos tiempos no se posiciona como la más expedita. En consecuencia, el legislador determinó la posibilidad de realizar otros procesos que cumplieran con necesidades determinadas y que, por su naturaleza, pudieran manejar algunas restricciones en la participación de los interesados dentro de los procesos de selección.

La modalidad de contratación directa se ha desarrollado en Colombia de manera restrictiva, determinando su aplicación para casos específicos, evitando consideraciones de factores subjetivos que alejen esta modalidad de los fines del Estado. Colombia, en su condi- 
ción de país en desarrollo, elaboró una regulación específica de la contratación bajo la modalidad de ciencia y tecnología, que llevan a cabo las entidades públicas con los particulares; lo que conllevó a la generación de normas que de manera clara buscaran reglar el proceso de contratación, y que permitieran que el Estado tuviera un grado de maniobrabilidad en estos casos específicos, con el fin de hacer más efectiva y transparente la contratación.

En este contexto surge la pregunta: ¿la modalidad de contratación directa bajo la causal de ciencia, tecnología e innovación se presenta como un mecanismo para que la administración pública pueda eludir el principio de planeación y de selección objetiva a partir de la Constitución Política colombiana de 1991? Ante la cual, a través de esta investigación se acoge una hipótesis negativa, que pretenderá demostrarse al identificar que, si bien la contratación directa bajo esta causal en principio no se acoge a los requisitos ni tiempos generales de la contratación pública, esta no está presupuestada para que se realice al libre arbitrio del administrador.

En esta investigación se desarrollará un objetivo general, que consiste en analizar si la contratación directa bajo la causal de ciencia, tecnología e innovación se presenta como un mecanismo para que la administración pública pueda evadir el principio de planeación y selección objetiva en la Constitución Política colombiana de 1991, a través de unos objetivos específicos, tales como l) delimitar el principio de planeación, en complemento con el principio de selección objetiva en el marco de la contratación estatal; 2) delimitar conceptualmente la contratación directa bajo la causal de Ciencia, Tecnología e Innovación, y 3) concluir el análisis de la aplicación de la causal de la contratación directa bajo el principio de selección objetiva.

Para el primer objetivo específico se realizará un análisis de doctrina relacionada con la descripción de los principios de planeación y de selección objetiva a partir de la Constitución Política de 1991, 
enmarcados dentro de la contratación pública; lo cual dará paso al segundo objetivo específico, a través del cual se desarrollará una revisión de doctrina y análisis de normatividad especifica de la contratación directa y de ciencia, tecnología e innovación, y así concluir con el tercer objetivo, donde a partir de la revisión legal y doctrinal de lo anteriormente planteado se llega a la conclusión de la validación de la hipótesis de este artículo.

Esta investigación es pertinente toda vez que en un país como Colombia la contratación pública está detalladamente reglada, y a través de los años se ha buscado una estructura fuerte que no permita al ejecutor tener la libertad de contratar sin hacer un proceso de selección objetiva. En este apartado, el autor pretende enfatizar la importancia de este estudio a partir de dos enfoques principales, que son a través de la doctrina y del análisis del estudiante para poder resolver la pregunta objeto de investigación.

La investigación se realizó con un enfoque hermenéutico-crítico, con un método de investigación cualitativo, desarrollado a través de una revisión documental, con el propósito de comprender los textos y dar validez y solución a los objetivos planteados en el trabajo, además de dar claridad al problema de la investigación.

Lo anterior favorece a la formulación de conclusiones que pretenden ayudar al lector de este documento académico a tener una visión frente a lo que significa la contratación directa bajo la causal de ciencia y tecnología, y, por lo tanto, a identificar claramente la seguridad jurídica que aplica al concepto del régimen especial de ciencia, tecnología e innovación en la contratación estatal colombiana. Por consiguiente, se comenzará haciendo la descripción del principio de planeación y su complementación con el principio de selección objetiva, la cual se hará desde los conceptos teóricos doctrinales en el marco de la contratación estatal como tema central, que incidirá para la escogencia de la modalidad de selección. 


\section{Delimitación del principio de planeación en complemento con el principio de selección objetiva en el marco de la contratación estatal}

En función de la introducción se procederá a realizar la delimitación del principio de planeación en complemento con el principio de selección objetiva, los cuales se revisarán dentro del marco de la contratación estatal, con el fin de desarrollar el primer objetivo planteado. Para esta delimitación se hará la revisión y análisis de la legislación colombiana y diferentes doctrinantes que se han pronunciado sobre los temas objeto de estudio para, de esta manera, enmarcar los conceptos dentro de la contratación estatal específicamente, que para efectos de esta investigación se tienen como las bases conceptuales de la misma.

En desarrollo de los fines del Estado que se establecen en la Constitución se encuentran los determinados para la contratación estatal dentro de la Ley 80 de 1993, que específicamente se refieren a que los servidores públicos considerarán, al celebrar contratos y en su ejecución, que las entidades buscan la continua y eficiente prestación de los servicios públicos y la efectividad de los derechos e intereses de los administrados que colaboran con ellas, en la consecución de dichos fines. Para el cumplimiento de estos fines se acude a los principios que se han descrito, como la obligación de que alguna acción sea realizada, en la medida de lo posible, dentro del marco de las realidades jurídicas (Alexy, 2010).

El artículo 209 de la Constitución Política de 1991 establece unos principios orientadores, como el de igualdad, moralidad, eficacia, economía, celeridad, imparcialidad y publicidad; mediante estos se busca establecer los presupuestos que sirven de guía en la ejecución de las funciones de los servidores públicos y se hace claridad sobre el hecho de que las entidades estatales están al servicio del interés general (Matallana, 2015, p. 65). Partiendo de los principios 
constitucionales, la Ley 80 de 1993 desarrolló en materia de contratación estatal unos puntos específicos para regular las actuaciones de quienes intervienen en la contratación estatal, tales como transparencia, economía y responsabilidad, que se encuentran de acuerdo con los postulados que rigen la función administrativa.

Al delimitar el principio de planeación en la selección objetiva de los contratistas desde la contratación estatal, se encuentra que este se presenta como base fundamental de la misma ya que a través de él se pretende que la administración tenga pleno conocimiento de la necesidad a suplirse y realice todas las acciones previas a una contratación; "para comprender la magnitud de este principio se debe observar que la planeación es un concepto propio del Estado social de Derecho, como criterio garantizador de legalidad y del interés general, pilares fundamentales dentro de la sociedad actual" (Expósito, 2003 , p. 122), es decir, que este principio permite que, después de un estudio por parte de la Administración, se tengan las reglas claras para los participantes.

El principio de planeación se encuentra establecido en el artículo 25 numeral 12 de la Ley 80 de 1993, modificado por el artículo 87 de la Ley 1474 de 2011, e indica que antes de realizar la apertura de un proceso de contratación se deben elaborar los documentos y estudios previos requeridos para el análisis, preparación y perfecto conocimiento de la necesidad de la entidad; es decir, que este principio se refiere a las actividades previas a un proceso que determinan la necesidad que se busca satisfacer de manera precisa y también a establecer temas como el objeto y los recursos con que se ejecutará el mismo. Todas estas acciones deben ser llevadas a cabo con anterioridad al inicio del proceso contractual (Amaya, 2016); entendiéndose que se refiere a

(...) los comportamientos que debe desplegar la Administración para conocer a profundidad el objeto a contratar y las diferentes opciones exis- 
tentes en el mercado; todo con el fin de optimizar los recursos y obtener la mayor efectividad y provecho respecto al negocio a celebrar, de modo que las circunstancias posteriores que puedan presentarse estén en lo posible dentro del campo de control del Estado para así tomar las medidas necesarias, reduciendo el terreno de la imprevisión a las situaciones realmente inesperadas dentro de una actitud diligente y consciente de la Administración Pública (Expósito, 2003, p. 604).

De esta manera, se pretende que, a través del principio de planeación, se realice el estudio juicioso de las posibilidades que existen en los mercados para suplir una necesidad del Estado; que se estructure un proceso con bases fuertes que identifique el objeto, los riesgos y las posibilidades de incumplimiento para evitarlas; asimismo, con el propósito de que en el cumplimiento de los fines del Estado se haga la selección que más le convenga a la Administración para la satisfacción de sus necesidades, se identifiquen la mayor cantidad de ofertas en el mercado.

En la contratación pública el principio de planeación se debe tomar como una guía en los aspectos procesales de la función de la Administración, los cuales son estudiados y seguidos durante las diferentes etapas de los procesos; el desarrollo de la misma va de la mano con los principios de transparencia y eficacia, pues a través de ellos se da la posibilidad de que los interesados conozcan los aspectos más relevantes de los procesos, evitando que queden eventos dejados a la improvisación (Amaya, 2016). Así las cosas, este principio responde claramente a la definición de principio como mandato optimizador del derecho (Bernal, 2005), pues debe aplicarse como rector en el desarrollo de todos los procesos contractuales.

A través de este principio se identifican las posibles complicaciones que puedan llegar a ocurrir durante el proceso, de manera tal que se pretendan evitar o, en caso de que sucedan, se determinen las acciones que se deben implementar para atenderlas, siendo esta "la 
primera etapa del proceso de contratación, mediante ella las entidades estatales viabilizan desde el punto de vista técnico y económico las obras, los bienes y servicios que se requieren para cumplir con los cometidos que les han encomendado" (Yong, 2013, p. 86); es decir, a partir de este estudio de la necesidad y del mercado, las entidades determinan desde diferentes perspectivas la pertinencia técnica, económica y jurídica del proceso.

El principio de planeación como se ha delimitado tiene como finalidad la transparencia y claridad en los procesos de contratación en busca de una selección objetiva para que los interesados puedan participar y se optimicen los recursos públicos. Al respecto, el legislador a través del artículo 5 de la Ley 1150 de 2007 estableció que es "objetiva la selección en la cual la escogencia se haga al ofrecimiento más favorable a la entidad y a los fines que ella busca, sin tener en consideración factores de afecto o de interés"; lo cual se logra con la definición previa y precisa de la necesidad y procedimiento para alcanzarla, estableciendo el deber para la Administración Pública:

(...) la tarea de realizar procedimientos de contratación basados en aspectos puramente objetivos, a efectos de evitar a toda costa el fenómeno de la corrupción administrativa, que las más de las veces se encontraba presente en dicha actividad pública y en que se desplazaba al interés general para anteponer consideraciones y aspectos de tipo subjetivo, particulares, a favor de los funcionarios que tenían la decisión de contratar (Expósito, 2003, p. 426).

Esta tarea se logra a través de la preparación del proceso, con la búsqueda de la satisfacción de una necesidad de la Administración, realizando el estudio del mercado y dejando claras desde el principio las reglas de participación en el proceso. La decisión del administrador a la hora de realizar una elección se encuentra determinada en la ley por los parámetros establecidos en el momento de iniciar un proceso en busca del interés general, es así como "el servidor público 
debe entonces mantenerse al margen de todas las consideraciones subjetivas para garantizar la selección correcta” (Benavides, 2010, p. 288). Por tal razón, el servidor debe seguir los criterios objetivos a los que se llega a través del análisis y la planeación de los procesos en todas sus etapas.

En la selección objetiva se exige que haya concurrencia de ofertas, para esto el artículo 2 de la Ley 1150 de 2007, determina las diferentes modalidades de procesos de contratación que se aplican para hacer la escogencia del contratista, como son la licitación pública, la selección abreviada, el concurso de méritos y la contratación directa, y para el desarrollo de las mismas se debe realizar un ejercicio previo que permita identificar cual es la modalidad que aplica para cada caso. Ninguna de estas modalidades puede realizarse sin que cumpla con el debido estudio de la necesidad para garantizar la libre competencia de los interesados, asegurada a través de la publicidad de los procesos.

Para desempeñar la función del Estado en desarrollo de la contratación estatal, se pretende cumplir con el principio de la selección objetiva que procura que los interesados en participar en los diferentes procesos de contratación tengan acceso en igualdad de condiciones y se optimicen los recursos públicos. El principio de la selección objetiva busca asegurar la igualdad de oportunidades y la selección del oferente, y para ello tiene diferentes manifestaciones (Castro, 2011). A través de este principio se pretende que el particular tenga las mismas condiciones al participar en un proceso, escogiendo el oferente que realiza la propuesta más favorable para la entidad y el cumplimiento de sus fines (Rodríguez, 2005); solo se busca que, mediante factores preestablecidos, se concrete la selección, sin hacer reparos en consideraciones de tipo subjetivo que puedan direccionar la escogencia del contratista.

Se encuentra en este punto con los sistemas creados por la ley encaminados a la escogencia de la oferta más favorable a los intereses 
de la entidad, regulados de manera expresa en la misma a partir de principios y normas de procedimiento de obligatorio cumplimiento por parte de la Administración, que aseguren la libertad de concurrencia de los particulares y una elección limpia del futuro colaborador en el logro de los fines mediatos e inmediatos del Estado (Expósito, 2013), para que de este modo se genere una seguridad jurídica en los procesos de la Administración y para el interesado en participar dentro de un proceso. La selección objetiva es concebida dentro del ordenamiento jurídico colombiano como un principio que, como se ha referenciado anteriormente, se establece como una norma de obligatorio cumplimiento para la Administración, así que:

el estatuto contractual colombiano, le da un sentido particular, en cuanto lo concibe como una norma rectora que se debe aplicar en los procesos de contratación estatal, por quienes la realicen y seleccionen o interpreten una norma para aplicarla a un caso relacionado con aspectos contractuales (Yong, 2013, p. 43).

Es así como se establecen reglas básicas en los principios de la contratación pública, desarrollados a través de los artículos 24 al 30 de la Ley 80 de 1993 (Molano, 2009), que son de obligatorio cumplimiento para la Administración en desarrollo de su función, siempre en busca de satisfacer las necesidades de la manera más idónea en pro del interés general, de asegurar la igualdad de oportunidades en la participación de los interesados y la selección del oferente más capaz. Esto se genera a través de dos manifestaciones, tales como la exigencia de establecer las reglas claras previamente y la que la adjudicación del proceso de selección se realice únicamente con base en esas reglas preestablecidas (Castro, García y Martínez, 2010), es decir, que los procesos tengan los criterios de selección determinados desde el inicio.

Se debe tener presente que los principios no son ajenos entre sí, ellos deben ser interpretados de manera conjunta y complemen- 
taria, puesto que son los orientadores de la actividad contractual de la Administración (Santofimio, 2010); es así como el principio de selección objetiva se analiza en conexión con el de planeación para el tema de estudio. Estos principios generan seguridad jurídica en los procesos, pues, como se encuentran establecidos, no son cambiantes, sino que sus enunciados deben ser tenidos en cuenta y cumplidos en las diferentes modalidades de selección, sin dar paso esto a que sean cumplidos en mayor o en menor medida dependiendo del caso en particular (Miranda, 2016). Están establecidos de manera general para que sean aplicados de igual forma en cada ocasión, $\mathrm{y}$ debido a su complementación se hacen necesarios e intrínsecos.

Así las cosas, se tiene que "la selección del contratista es uno de los aspectos más importantes dentro de la contratación del Estado, por cuanto su aplicación deriva en un resultado que no depende de la simple voluntad de la Administración contratante" (Expósito, 2013, p. 55), sino de los procesos de planeación realizados previamente, en donde se hace el análisis de la modalidad de selección y se permite que haya una igualdad frente a la postulación de propuestas por parte de los oferentes, de manera tal que la Administración pueda seleccionar la oferta que cumpla con todos los requisitos y sea la más conveniente, sin que intervengan para esto factores subjetivos.

Se ha identificado la licitación pública como la modalidad de selección objetiva por excelencia, debido a que esta es la que permite el mayor número de participación de interesados al proceso, y tiene la característica que es un procedimiento ampliamente reglado que logra limitar la libertad de la administración (Benavides, 2004, p. 293); por lo tanto, en principio se concibe como la modalidad más favorable para el cumplimiento de los fines del Estado, al permitir realizar evaluaciones de diferentes propuestas, determinándose así que existe mayor probabilidad para que la Administración optimice sus recursos y contrate con el mejor oferente. 
Sin embargo, no se puede establecer que la pluralidad de oferentes, en principio, sea la única forma de permitir la selección objetiva, pues en algunos casos la escogencia podría realizarse con la participación de un oferente, es decir, que para escoger la oferta más favorable la entidad debe observar las reglas que dispone la ley (Fandiño, 2014), y esto no siempre se cumple en la presentación de una gran cantidad de ofertas, más aún cuando la necesidad que debe suplirse necesita ejecutarse a través de un proceso ágil, lo que, por la especificidad de esta, no da lugar a la participación de un gran número de oferentes.

A través de los principios de planeación y de selección objetiva en las diferentes etapas de los procesos de contratación se pretende que los criterios de selección de los contratistas sean objetivos y reglados sin dar oportunidad a los administradores a incluir criterios subjetivos que puedan direccionar las contrataciones. Una vez identificados y analizados, se procederá a enmarcar el concepto de contratación directa y su desarrollo bajo la causal de Ciencia, Tecnología e Innovación, que surge a partir de la planeación de los procesos y su estructuración, con el fin de conseguir el ofrecimiento más favorable para la entidad a través del cual se pueda satisfacer su necesidad.

\section{Delimitación conceptual de la contratación directa bajo la Causal de Ciencia Tecnología e Innovación y su aplicación en el principio de selección objetiva}

En esta sección se realizará la delimitación conceptual de la contratación directa bajo la causal de ciencia tecnología e innovación a partir de su aplicación en el principio de selección objetiva, la cual se da mediante la planeación de los procesos. Por lo cual, se pretenden identificar los criterios con los que debe cumplir la Administración al momento de realizar esta modalidad de contratación, toda vez que, como se verá a lo largo de este capítulo, es una causal plena- 
mente reglada que goza de formalidades, como cualquier otra modalidad de contratación, pero que son más expeditas.

La Administración debe identificar su necesidad con el propósito de dar aplicación a los principios de la contratación y dar estricto cumplimiento a los fines del Estado y de la contratación misma. Razón por la cual el legislador ha propendido por la delimitación de las posibilidades para el ejecutor de realizar los procesos de manera tal que la elección del contratista no esté sujeto a la mera liberalidad de la voluntad de la Administración. Entonces, "tanto la doctrina como la jurisprudencia y el legislador se esfuerzan por reducir el carácter discrecional de la selección, con frecuencia asimilada a selección arbitraria” (Benavides, 2004, p. 309), generando límites legales y procedimientos específicamente establecidos para tal fin, el cual debe cumplir el ejecutor en el caso que pretenda hacer uso de este tipo de modalidad.

Para esta investigación se hará énfasis en la modalidad de contratación directa que, si bien se ha planteado como un procedimiento más ágil de contratación, debe cumplir con unos requisitos básicos impuestos por el legislador, con el fin de generar unas restricciones a la libertad de la Administración. "El peso de las reglas presupuestales y la autorrestricción de la competencia a través de la reglamentación de los procedimientos de selección de contratistas reflejan una voluntad de restringir toda libertad" (Benavides, 2004, p. 319); es decir, se pretende limitar al máximo la capacidad y posibilidad del ejecutor al momento de realizar la escogencia del contratista que va a suplir la necesidad de la Administración, lo cual denota la desconfianza que se le tiene a la misma por parte del primero.

Aunque se ha descrito que la contratación directa es "el procedimiento por entendimiento directo en el que la entidad pública previo el (análisis) cumplimiento de requisitos menos formales, como también el acaecimiento de ciertas situaciones o circunstan- 
cias, elige al contratante" (Solano, 1994, p. 146), esta definición ha sido criticada, pues se indica que aunque la contratación directa no es un proceso concursal, para realizar la escogencia del contratista se deben cumplir los requisitos formales de la contratación, es decir, se deben aplicar los principios y se debe seleccionar con objetividad al contratista (Expósito, 2003). Por tal razón, no se considera que se pueda indicar como tal que los requisitos sean menos formales, pues están determinados y son de obligatorio cumplimiento, sino que por la necesidad misma que debe suplirse estos procedimientos están concebidos para que sean más expeditos.

Es preciso resaltar que la contratación directa no está determinada para ser la regla general; desde un principio se ha establecido como una excepción. Dentro de la regulación colombiana el legislador señaló las causales estrictas y explícitas en donde era aplicable la modalidad de contratación directa, así como el procedimiento para la escogencia y justificación de la referida causal, atendiendo a lo dispuesto en el artículo 87 de la Ley 1474 de 2011. De esta manera se genera una reserva legal, describiendo las situaciones específicas en las que se puede aplicar esta contratación y el administrador no puede ampliar estos criterios ni usarlos por similitud (Araujo, 2011). Esta causal debe estar identificada a través del estudio y análisis previo de la contratación y debe cumplir con unos criterios específicos.

Los estudios previos se elaboran para justificar el tipo de contratación que se debe seguir y de esta forma determinar si se ajusta a las causales establecidas por el legislador, con el fin de salvaguardar el principio de planeación con el que se garantizó la modalidad de contratación que soporte el contrato (Giraldo y Giraldo, 2018), esto teniendo en cuenta que "en las etapas precontractual y contractual, las autoridades públicas deben dar aplicación obligatoria y estricta a los principios constitucionales, legales, e incluso los no regulados por el legislador, aplicables al contrato Estatal” (Vidal, 2008, p. 39), 
buscando siempre mantener la legalidad y el bien común con los que pretende cumplir la Administración.

Para el legislador no solo bastó con la realización de unos estudios que determinaran la necesidad específica y la descripción del procedimiento, sino que exigió la realización de un acto administrativo por parte del ordenador del gasto que soportara y justificara la elección de esta modalidad de contratación y la razón para que no se acudiera a un proceso licitatorio o a otra modalidad de contratación; esto se encuentra regulado en el artículo 2.2.1.2.1.4.1 del Decreto 1082 de 2015. Dentro de este acto se establece información sobre la causal que se invoca para la contratación, el objeto de lo que se va a contratar, el valor y obligaciones del contratista y demás información sobre el lugar de consulta de los documentos previos.

Ahora bien, el país a partir de la Constitución Política de 1991 ha dejado de ser un Estado proteccionista y ha buscado incluir más a las empresas nacionales en el desarrollo de sus funciones, por lo que se han adoptado varias reformas en la normatividad para establecer marcos regulatorios claros y transparentes, es decir, más predecibles, con el fin de dar mayor seguridad a los particulares y de esta manera aumentar su participación en el desarrollo de los fines del Estado (OCDE, 2013). En 1991 se determinó la necesidad del Estado de promover la investigación y la ciencia en el país, pero para lograr esto debió regularse a través de legislación específica que definiera las actividades de ciencia y tecnología, que conllevaran a la celebración de contratos especiales:

contratos de ciencia y tecnología. Son los que tienen por objeto la investigación científica y tecnológica con el fin de producir conocimientos. Se aplica cuando el objeto sea la transferencia o uso de productos de la ciencia o la tecnología. Las características de este tipo de contratos se encuentran en el Decreto- ley 591 de 1991 (Pachón, 2014, p. 124). 
Esto está establecido por las políticas estatales colombianas, y se encuentra unido con la necesidad de una contratación eficiente y que, a su vez, pueda garantizar el resguardo de los principios de la contratación estatal y del Estado, pero que logre que la adquisición que se requiera sea rápida y genere provecho para el avance del país, teniendo siempre presente que los recursos que son la parte esencial de la contratación son estatales. Lo cual se logra a través de la contratación directa, siendo este un procedimiento que se precia por ser ágil, y dentro del cual no se exige el agotamiento de diferentes etapas del proceso general de contratación (Rosero, 2012), sin dejar de ser un procedimiento regulado que establece el cumplimiento de formalidades y análisis previo.

Es decir, que para la aplicación de este tipo de modalidad de contratación la ley ha determinado unas características detalladas que no permiten que sea aplicable a menos que se cumplan los presupuestos precisados en la ley específicamente; por tal razón, no aplica la conexidad o accesoriedad, lo que evita que la decisión de contratar bajo la causal de desarrollo de ciencia, tecnología e innovación quede al libre arbitrio del ejecutor, lo cual se conoce a partir del proceso de "formación de la voluntad de la Administración inicia con la etapa de planeación, la cual constituye un principio y un deber a cargo de los servidores públicos” (Fernández, 2016, p. 41), etapa a través de la cual se realiza el análisis de la necesidad y las incidencias que puedan ocurrir durante el desarrollo del proceso con el fin de establecer claramente la modalidad de selección a ejecutar.

Así las cosas, para realizar la contratación a través de la modalidad de contratación directa mediante la causal de desarrollo de ciencia y tecnología se debe cumplir con unos criterios específicos, es decir, que además de hacer observancia a los principios constitucionales y de contratación, las actividades a contratar deben estar incursas en lo dispuesto en la normatividad para el desarrollo de las actividades de ciencia, tecnología e innovación, las cuales fueron regladas a partir 
del año 1990 con la Ley 29 de $1990^{2}$, la cual fue desarrollada inicialmente por el Decreto 393 de $1991^{3}$ y por el Decreto Ley 591 de $1991^{4}$, en donde el legislador estableció lo que se debe entender como actividades científicas y tecnológicas y el procedimiento para la contratación de bienes y servicios que tengan que ver con su desarrollo.

Dentro de la normatividad colombiana no se encuentran descritos los conceptos de ciencia, tecnología e innovación, específicamente, debido a que estos son criterios que están en constante evolución y no son específicamente criterios jurídicos, sino que incluyen otras ciencias; no obstante lo anterior, el legislador en 1991, mediante el decreto que reguló lo relacionado con las modalidades de contratos que sirvieran para el fomento de actividades científicas y tecnológicas, estableció una definición para estas actividades. Por su relevancia es necesario enunciar lo que se entiende por ellas:

1. Investigación científica y desarrollo tecnológico, desarrollo de nuevos productos y procesos, creación y apoyo a centros científicos y tecnológicos y conformación de redes de investigación e información. 2. Difusión científica y tecnológica, esto es, información, publicación y divulgación y asesoría en ciencia y tecnología. 3. Servicios científicos y tecnológicos que se refieren a la realización de planes, estudios, estadísticas y censos de ciencia y tecnología; a la homologación, normalización, metodología, certificación y control de calidad; a la prospección de recursos, inventario de recursos terrestres y ordenamiento territorial; a la promoción científica y tecnológica; a la realización de seminarios, congresos y talleres de ciencia y tecnología, así como a la promoción y gestión de sistemas de calidad total y de evaluación tecnológica 4. Proyectos de innovación que

2 Ley 29 de 1990. "Por la cual se dictan disposiciones para el fomento de la investigación científica y el desarrollo tecnológico y se otorgan facultades extraordinarias".

3 Decreto 393 de 1991. "Por el cual se dictan normas sobre asociación para actividades científicas y tecnológicas, proyectos de investigación y creación de tecnologías".

4 Decreto 591 de 1991. "Por el cual se regulan las modalidades específicas de contratos de fomento de actividades científicas y tecnológicas". 
incorporen tecnología, creación, generación, apropiación y adaptación de la misma, así como la creación y el apoyo a incubadoras de empresas, a parques tecnológicos y a empresas de base tecnológica. 5. Transferencia tecnológica que comprende la negociación, apropiación, desagregación, asimilación, adaptación y aplicación de nuevas tecnologías nacionales o extranjeras. 6. Cooperación científica y tecnológica nacional e internacional (Decreto 591 de 1991, art. 2).

A través de las actividades definidas en este decreto, el legislador buscó dar un cierre a la posible interpretación de la causal, haciendo una descripción de lo que se considera actividades de ciencia y tecnología, sin dar campo a la interpretación de la Administración; es decir, el legislador dio pautas muy precisas y supuestos normativos muy concretos para que pueda operar (Araújo, 2011) la modalidad de contratación directa bajo la causal de ciencia, tecnología e innovación. Por tal razón, no se puede acudir únicamente a lo establecido en la ley general, sino que esta se debe aplicar en conjunto con lo establecido en las disposiciones específicas que regulan la materia y, a su vez, no permiten la interpretación por parte del ejecutor.

Ahora bien, el Estado colombiano, en vista de la importancia de la investigación a nivel mundial, identificó la necesidad de mejorar sus procesos para la contratación de actividades específicas en el campo de la investigación de ciencia y tecnología, pretendiendo que las entidades, las empresas y las universidades trabajasen bajo un mismo marco jurídico en las mencionadas actividades, para que en el momento de contratar con el Estado esto sea posible realizarlo de manera más efectiva, debido a que la relación de este con los particulares debe adelantarse de manera reglada, dando aplicación a los principios de la contratación estatal (Castro, García y Martínez, 2010). Esto genera una seguridad jurídica para el particular al hacer que los procesos sean claros y desde sus etapas iniciales se encuentren definidos en su totalidad; de igual forma se ha establecido que la acción del Estado debe estar focalizada en 
(...) el desarrollo de sectores estratégicos en el largo plazo, que se caractericen por la producción de bienes y servicios de alto contenido científico y tecnológico y, por ende, de alto valor agregado. Esta focalización es necesaria para alcanzar alto impacto y evitar dispersión de recursos y se materializará a través de priorizar en los instrumentos y programas las áreas estratégicas de energía y recursos naturales, biotecnología, salud, materiales y electrónica, tecnologías de información y comunicaciones, logística y diseño y construcción de ciudadanía e inclusión social (Departamento Nacional de Planeación, 2009).

Lo anterior se da toda vez que el Estado tiene la titularidad y la obligación de promover y fomentar la ciencia y la tecnología, que se manifiesta en unas actividades específicas, como son el fortalecimiento de la investigación científica, la promoción y fomento del acceso a la ciencia, la inclusión de recursos para el desarrollo de las ciencia en los planes de desarrollo, así como la creación de incentivos para personas e instituciones que desarrollen y fomenten la ciencia y la tecnología y el ofrecimiento de estímulos especiales a personas e instituciones que ejerzan actividades científicas y tecnológicas (Chávez, 2012); es decir, que el Estado se encuentra encargado en generar las condiciones para el desarrollo de la ciencia en el país y para esto debe garantizar que los procesos sean claros y ágiles fomentando movimiento en la contratación del Estado con la participación de empresas particulares.

A través de las diferentes leyes se ha buscado hacer claridad en temas específicos del régimen aplicable a los contratos para desarrollar las actividades de ciencia y tecnología y lo que deben tener en cuenta las entidades que pretendan realizar este tipo de contratación independientemente de la fuente de los recursos financieros, es decir, que la entidad estatal, previa la revisión y estudio de su necesidad, debe determinar si las actividades que va a contratar se enmarcan dentro del catálogo que establece el legislador para las actividades de ciencia, tecnología e innovación (Agencia Nacional de Contratación Pública, 
2019). Esto se encuentra igualmente descrito en el artículo 33 de la ley 1286 de 2009, en donde establece específicamente que para el desarrollo de las actividades de ciencia, tecnología e innovación a través de convenios o contratos se rigen por la normas especiales y su procedimiento se efectúa a través de la contratación directa.

Analizado lo anterior, se ha establecido que, para que la Administración pueda hacer uso de la modalidad de contratación directa bajo la causal de ciencia, tecnología e innovación, no basta simplemente con hacer una justificación y análisis, sino que esta decisión se debe enmarcar en las actividades plenamente identificadas en la normatividad y dar cumplimiento a los procedimientos establecidos para este tipo de contratación, que si bien pueden ser ágiles, esto no quiere decir que restrinjan la aplicación del principio de selección objetiva. El legislador determinó las actividades de ciencia, tecnología e innovación, con lo que se establecieron los parámetros detallados para acceder a este tipo de contratación.

\section{Conclusiones}

Después de la revisión normativa y doctrinal realizada en las secciones precedentes, se delimitaron los conceptos de planeación, selección objetiva y contratación directa bajo la causal de ciencia, tecnología e innovación dentro del marco de la contratación estatal en Colombia, que es un punto neurálgico de la actividad del Estado.

En la primera sección se desarrolló el concepto de planeación, a través del cual se obliga a la Administración a realizar todos los procesos previos de análisis del requerimiento, el camino para concretarlo y los medios idóneos mediante los cuales se llega a la consecución de este. Por otro lado, se destacó que la selección objetiva busca que la elección de la persona que vaya a contratar con el Estado se haga después de un proceso reglado y claro desde el 
inicio. Se identificó que los principios de planeación y de selección objetiva se interrelacionan y se complementan en el desarrollo de los procesos contractuales bajo la modalidad de contratación directa, ya que al aplicarlos permiten que desde la fase inicial se cuente con una seguridad jurídica, generando que los procesos sean transparentes tanto para los participantes como para la Administración.

Al desarrollar la segunda sección, se conceptualizó la modalidad de selección por contratación directa como un procedimiento más ágil en el momento de realizar la contratación, sin ser un procedimiento que no cumpla las formalidades necesarias para garantizar la selección objetiva de los contratistas del Estado. Esta modalidad de contratación fue concebida por el legislador como una excepción para aplicarse en situaciones específicas que ameritaran una celeridad, que no era posible mediante el proceso de licitación.

Dentro de la contratación directa como proceso de selección excepcional se estudió la causal de ciencia, tecnología e innovación, a través de la cual la Administración realiza la selección del contratista sin hacer un proceso público en el que participen los posibles interesados, como resultado del análisis del Decreto 591 de 1991, en el cual se establecen taxativamente las actividades que hacen parte de esta causal, limitando al ejecutor en la aplicación de la misma. El estudio de la elección de esta modalidad de contratación no da campo a que el ejecutor interprete cuales son las actividades, por tal razón, el análisis no puede ser extensivo.

Ante la pregunta planteada para desarrollar en este estudio: ¿la modalidad de contratación directa bajo la causal de ciencia, tecnología e innovación se presenta como un mecanismo para que la administración pública pueda eludir el principio de planeación y de selección objetiva a partir de la Constitución Política colombiana de 1991?, a través de la revisión y el análisis se ha identificado que para hacer uso de esta modalidad, la Administración debe aplicar 
unos criterios específicos que fueron regulados por el legislador para tal fin.

Por lo anterior, el resultado de esta investigación es que se avala la hipótesis planteada como desarrollo a la pregunta. Como se ha establecido, la Administración no puede acudir a la modalidad de contratación directa bajo la causal de ciencia, tecnología e innovación sin el análisis enmarcado en lo establecido taxativamente por el legislador y cumpliendo las respectivas formalidades propias de los procesos de selección por parte del Estado; por lo tanto, la aplicación de estas actividades no dan lugar a interpretación o analogía. Como consecuencia, la Administración está en la obligación de hacer una revisión profunda de las formalidades y procedimientos al contemplar esta modalidad.

Sin embargo, después de la revisión realizada, no se pueden desconocer los riesgos inherentes a los conceptos que se deben tener en cuenta como planteamiento de un panorama completo. Se identificó que dentro de la legislación no se encuentran definidos los conceptos de ciencia, tecnología e innovación específicamente, lo que ha generado para la Administración dificultades al emplear esta causal bajo la modalidad de contratación directa, pues los ejecutores tienden a desconocer e interpretar erróneamente lo dispuesto en el artículo 2 del Decreto 591 de 1991, generando confusión en su aplicación.

Adicionalmente, se identificó que los conceptos de ciencia, tecnología e innovación están presentes en los diversos ámbitos de las actividades del Estado y se encuentran en constante evolución; por lo tanto, analizados a la luz de la normatividad colombiana se considera que la norma que describe estas actividades al ser un decreto de 1991 no abarca a cabalidad los temas que se desarrollan en la actualidad al respecto y que demandan especial atención de un estado moderno. 


\section{Referencias}

Agencia Nacional de Contratación Pública (2013). Circular externa No. 6 del 27 de septiembre de 2013. Agencia Nacional de Contratación Pública - Colombia Compra Eficiente. https://www.colombiacompra.gov.co/sites/cce_public/files/ cce_circulares/20130927circularcit.pdf

Agencia Nacional de Contratación Pública (2019). Circular Externa Única. Agencia Nacional de Contratación Pública - Colombia Compra Eficiente https://www.colombiacompra. gov.co/sites/cce public/files/cce circulares/cce circular unica.pdf

Alexy, R. (2010). Teoría de los Derechos Fundamentales (Segunda edición en castellano). Centro de Estudios Políticos y Constitucionales.

Amaya, C. F. (2016) El principio de planeación en la contratación estatal, un principio no tipificado. Revista Via luris, (20), 105-119.

Araújo, R. (2011). La Ley 1150 de 2007. ¿Una respuesta a la eficacia y transparencia en la contratación estatal? Universidad del Rosario.

Bernal, C. (2005). El Derecho de los derechos. Universidad Externando de Colombia.

Benavides, J. L. (2010). El Contrato estatal. Entre el derecho público y el derecho privado (Segunda edición). Universidad Externado de Colombia.

Castro C. (2011). Transparencia y lucha contra la corrupción en la contratación estatal, en la ley 1150 de 2007, una respuesta a la eficacia y trasparencia en la contratación estatal la nueva contratación pública en Colombia. Universidad del Rosario.

Castro, C., García, L. F. y Martínez, J. R. (2010) La contratación estatal. Teoría general. Perspectiva comparada y regulación internacional. Universidad del Rosario.

Chávez, A. R. (2012). Los convenios de la administración: entre la gestión pública y la actividad contractual. Universidad del Rosario.

Constitución Política de Colombia (1991). (2a ed.) Gaceta Constitucional No. 116 de 20 de julio de 1991.

Decreto-Ley 393 de 1991. Por el cual se dictan normas sobre asociaciones para actividades científicas y tecnológicas, proyectos de investigación y creación de tecnologías. 8 de febrero de 1991. DO. N. . 39672. 
Decreto No. 591 de 1991. Por el cual se regulan las modalidades específicas de contratos de fomento de actividades científicas y tecnológicas. 26 de febrero de 1991. D0. N.․ 39702 .

Decreto 1082 de 2015. Por medio del cual se expide el decreto único reglamentario del sector administrativo de planeación nacional. 26 de mayo de 2015. D0. N. ․ 49523.

Departamento Nacional de Planeación (2009). Documento CONPES 3582. Consejo Nacional de Política Económica y Social República de Colombia Departamento Nacional de Planeación. https://minciencias.gov.co/sites/default/files/upload/reglamentacion/ conpes-3582-2009.pdf

Expósito, J. C. (2003). La configuración del contrato de la administración pública en derecho colombiano y español. Universidad Externado de Colombia.

Expósito, J. C. (2013). Serie Derecho Administrativo No. 19 Forma y contenido del Contrato Estatal. Universidad Externado de Colombia.

Fandiño Gallo, J. E. (2014). La Contratación Estatal. Leyer.

Fernández, M. (2016). Fundamentos contemporáneos del derecho público: transformaciones necesarias. Universidad Católica de Colombia.

Giraldo Quintero Valencia, R. y Giraldo Valencia, E. 0. (2018). Violación de principios constitucionales y legales en el contrato No. 638 de 2013. Summa luris, 6(1), 159-182.

Ley 29 de 1990. Por la cual se dictan disposiciones para el fomento de la investigación científica y el desarrollo tecnológico y se otorgan facultades extraordinarias. 27 de febrero de 1990. DO. N. ․ 39205.

Ley No. 80 de 1993. Por la cual se expide el Estatuto General de Contratación de la Administración Pública. 28 de octubre de 1991. D0. N. ․ 41094.

Ley 1286 de 2009. Por la cual se modifica la Ley 29 de 1990, se transforma a Colciencias en Departamento Administrativo, se fortalece el Sistema Nacional de Ciencia, Tecnología e Innovación en Colombia y se dictan otras disposiciones. 23 de enero de 2009. D0. N. . 47241.

Matallana, E. (2015). Manual de Contratación de la Administración Pública (Cuarta Edición). Universidad Externado de Colombia.

Miranda, M. (2016). La regla 0 el principio de La selección objetiva en La contratación pública colombiana. Revista Verba luris, 11(35), 65-84. 
Molano, M. (2009). La nueva estructuración de los procesos selectivos en la contratación estatal. Ediciones Nueva Jurídica.

OCDE (2013). Public procurement in Colombia, en Colombia: Implementing Good Governance. OECD Publishing. http://dx.doi.org/10.1787/9789264202177-10-en

Pachón, C. (2014). Contratación pública, análisis normativo, descripción de procedimientos. Eco Ediciones.

Rodríguez, L. (2005). Derecho Administrativo General y colombiano. Temis.

Rosero, B. (2012). Contratación estatal. Manual teórico-práctico. Ediciones de la U.

Santofimio Gamboa, J. 0. (2010). Contratación estatal legislación. Universidad Externado de Colombia.

Solano, J.E. (1994). Contratación administrativa, Ley 80 de 1993. Comentarios legislacióndoctrina-jurisprudencia. Librería del Profesional.

Vidal, J. (2008). Derecho Administrativo. Editorial Legis.

Yong, S. (2013). El Contrato Estatal en el Contexto de la Nueva Legislación. Ibáñez. 